von der Fußstufe bis in große Höhe. Im Nacheinander starteten sechs Teilunternehmungen, im März 1964 folgte eine siebente. Zwei weitere Expeditionsgruppen zu völkerkundlichen Aufnahmen sollen folgen. Die erheblichen Kosten bestritt die Fritz-Thyssen-Stiftung. Die beigegebenen Abbildungen vermitteln einen Überblick über die eindrucksvolle Landschaftsszenerie. Spezielle Veröffentlichungen werden folgen, und zwar neben den Textbänden eine Zweiblätter-Karte 1:50000 auf Grund terristrischer Photogrammetrie, die zugleich Lücken zwischen älteren Aufnahme- und Untersuchungsgebieten schließt. Die angestrebte Monographie wird ein Standardwerk der Hochgebirgsforschung sein. Die vergleichende Heranziehung des Kenya-Gebietes kennzeichnet einen Grundzug moderner Erdraumforschung, die im Grunde eine landschaftsökologische und kulturlandschaftliche genannt werden kann. Praktisch wird die vielgliedrige Unternehmung von den Beteiligten als Grundlageforschung zur Entwicklung des Landes Nepal angesehen. Bei den ersten entsprechenden Finanzierungsversuchen waren allerdings die hierfür maßgebenden deutschen Dienststellen für diesen Aspekt nicht zu gewinnen. WILLI CZAJKa (Göttingen)

Trimborn, Hermann: Die indianischen Hochkulturen des alten Amerika. (Verständliche Wissenschaft, Bd. 82.) Berlin-Göttingen-Heidelberg: Springer 1963. VIII, 156 S. u. 57 Textabb. Kl.-80. Kart. DM 7,80 .

Die indianischen Hochkulturen ordnen sich in räumlichem Zusammenhang den stark gegliederten Gebirgslandschaften von Mexiko bis zum zentralen Anden-Hochland zu. Sie wuchsen aus einem nordsüdlichen Wanderungsproze $\beta$ hervor. Andererseits ist die Ausbreitung der Metalltechnik einer entgegengesetzt gerichteten Kulturübertragung zu verdanken Das Buch mußte daher bei aller Berücksichtigung der regionalen Besonderheiten sowohl implizit wie abschließend den Gesamtzusammenhang aufzeigen. Die hypothetische Erörterung möglicher Einzeleinflüsse direkt über den Pazifik wirc weitgehend vermieden, so daß eine Fülle von Tatsachen ausgebreitet werden kann. Dadurch werden die entworfenen Kulturbilder methodisch begründbar, soweit es der Raum des handlichen Bandes gestattet. Die Quellenlage ist spezifisch Die archäologische Beobachtung, auch des Kalenders, und die Kenntnis der heutigen Nachfahren, die kulturell allerdings abgesunken sind, schieben sich gegenüber den Aussagen der direkten Überlieferung in den Vordergrund. Die vorspanischen Kulturen waren nicht nur regional sehr verschieden, sondern von unterschiedlicher Höhe auf den Teilgebieten der Gesamtzivilisation. Die gewählte Textabfolge von Nord nach Süd in fünf räumlichen Abschnitten von Alt-Mexiko bis Groß-Perú ist nicht nur dem Wanderungsvorgang immanent, sondern dürfte die übersichtliche Darbietung der Forschungsergebnisse am besten ermöglichen. Der Gebirgscharalster ließ schon in der Vergangenheit neben dem Landverkehr dem Seeweg große Bedeutung zukommen. Die in den Herrschaftssystemen sich ausprägende Raumorganisation ist militärisch und ständisch jeweils von verschiedenem Gewicht. Indem so die Lagebeziehungen voll zur Geltung kommen, ist das Buch nicht nur als Darstellung der Hochkulturen sehr willkommen, sondern verdient auch wegen der Erschließung grundsätzlicher Einsichten aus der archäologischen und ethnologischen Forschung weite Beachtung. Ausgewählte Literaturangaben und Register sind angefügt.

WiLli CZAJKA. (Göttingen)

Soviet Antarctic Expedition. Information Bulletin. Vol. I und II. Amsterdam-London-New York: Elsevier Publ. Comp.
1964. Vol. I: XV, 404 S., 105 Abb., 53 Tab. Gr.-80. Dfl. 45,- (DM 50,-). Vol. II: X, 318 S., 108 Abb., 64 Tab. Gr.-80 Dfl. $40,-($ DM $45,-$ ).

Die neue Periode internationaler Antarktisforschung seit 1956 hat eine solche Fülle von Beobachtungen zur Geophysik (einschließlich Meteorologie und Ozeanographie), Glaziologie, Geologie, aber auch Biologie des Südpolargebietes erbracht, daß die Orientierung über die Art der Forschungsprogramme und den Stand ihrer Durchführung zu einem Problem für sich geworden ist. Da es oft lange dauert, bis die ausführlichen Ergebnisse im Druck erscheinen können, ist die Unterrichtung der wissenschaftlichen Welt durch rasche Veröffentlichung vorläufiger Berichte notwendig, nicht zuletzt um dadurch auch die weitere Planung zu erleichtern. Diesen Zweck erfüllen Informationsbulletins, die von allen an der Erforschung der Antarktis beteiligten Nationen herausgegeben werden, seit 1958 auch von der Sowjet-Union, die einen hervorragenden Anteil an der abschließenden Phase der Antarktisforschung hat. Die Ergebnisse dieser bedeutenden Expeditionen, die wesentliche Beiträge zur Kenntnis der Ostantarktis darstellen, sind bisher wegen der Sprachschwierigkeiten in der westlichen Welt nicht gebührend beachtet worden.

Die vorliegende sehr verdienstvolle englische Übersetzung geht auf die Initiative von GEORGE P. Woollard, Direktor des Geophysical and Polar Research Center, University of Wisconsin, Madison, Wisc., USA zurück. Band I enthält 130 Artikel aus den Bulletins Nr. 1 bis 10 (1958 bis 1959), Band II 84 Artikel aus den. Bulletins Nr. 11 bis 20 (1959 bis 1960), Band III ist in Vorbereitung. Obwohl es in dieser kurzen Besprechung nicht einmal möglich ist, die wichtigsten Titel anzuführen, ist eine Statistik der behandelten Wissensgebiete aufschlußreich. Von den insgesamt 214 Artikeln entfallen auf die Biologie 42, Glaziologie 40, Meteorologie 35, Geologie und Seismik 27, Ozeanographie 25, Geodäsie, Gravimetrie und Geographie 17, Polarlicht und Erdmagnetismus 13, Zusammenfassende Berichte über die Expeditionen 10, Navigation, Radioverkehr und Transport 5.

Das Werk macht somit vielen Interessenten aus dem weiten Kreis der Geowissenschaften wertvolle Informationen leicht zugänglich, zumal jeder Band einen Index nach Sachgebieten und Namen enthält. Der vermittelte Eindruck einer erstaunlichen Aktivität sowjetischer Forscher in der Antarktis wird noch gesteigert durch die in den meisten Bulletins vorhandenen bibliographischen Angaben über weitere Antarktisliteratur aus der Sowjet-Union. Die zahlreichen Abbildungen und Tabellen sind eine wertvolle Ergänzung der knapp und leicht verständlich geschriebenen Texte. Es muß allerdings berücksichtigt werden, daß die Artikel zwischen 1958 und 1960 erschienen sind und daß einige davon den Charakter vorläufiger Berichte haben, wobei sich gelegentlich Revisionen als notwendig erwiesen; so wurde z.B. die Höhe des Felssockels unter der Station Vostok in Band II, S. 5, mit 2150 m angegeben, im gleichen Band II, S. 249, aber auf Grund neuer Messungen auf $-300 \mathrm{~m}$ korrigiert. Ähnliche Interpretationsschwierigkeiten haben sich natürlich auch bei den seismischen Sondierungen anderer Expeditionen ergeben. Dieses Beispiel soll lediglich die kritische Art der Berichterstattung charakterisieren und zeigen, wie Schritt für Schritt die Kenntnis der wahren Natur der Antarktis mühsam angenähert werden muß. Die vorliegenden Bände enthalten so zahlreiche und wertvolle Beiträge hierzu, daß jeder, der sich ernsthaft für das Südpolargebiet interessiert, sie zur Kenntnis nehmen muB.

H. HoINKes (Innsbruck)

\title{
Berichtigungen und Bemerkungen
}

zu der Kurzen Originalmitteilung von W. MADER, H. HorN und R. ZIEGELBEcker [Naturwissenschaften 52, 55 (1965)] Der Titel der Arbeit enthält einen irreführenden Schreibfehler; er muß lauten: ,Identifizierung nadelförmiger Wolfram- und Wolframkarbideinkristalle in Pulvern".

zu der Kurzen Originalmitteilung ,Entsalzung von Huminsäuren an Sephadex G-25“ von R. Obenaus und H.-J. NeumanN [Naturwissenschaften 52, 131 (1965)]: In der Legende zu Fig. 1 sind die Zeichenerklärungen vertauscht worden. Es muß heißen: - Huminsäuren (HS); •—— Natriumchlorid ( $\mathrm{NaCl}$ )

zu der Kurzen Originalmitteilung ,Sind occipitale Alphawellen (,EEG-Muster“) sprachakustischer Art?“ von K. BiER Naturwissenschaften 51, 561 (1964)]: Der Redaktion ist vom Städtischen Kinderkrankenhaus in München-Schwabing erklärt worden, daß die Arbeit von Frau Dr. K. BIER ohne Wissen und Zustimmung der ärztlichen Leitung des Krankenhauses veröffentlicht worden ist. 